\title{
OPTICAL AND ELECTROCHEMICAL STUDY OF THE SULPHIDE/SILVER SYSTEM
}

\author{
S. Juanto, R. O. Lezna and A. J. Arvin \\ INIFTA, Facultad de Ciencias Exactas, Universidad Nacional de La Plata, Sucursal 4, \\ Casilla de Correo 16, La Plata (1900), Argentina
}

(Received 8 February 1993; in revised form 9 June 1993)

\begin{abstract}
Abatract-The behaviour of suphide species at a silver/electrolyte interface along with the electrodeposition of thin $\mathrm{Ag}_{2} \mathrm{~S}$ films have been studied by a combination of electrochemical and in situ optical methods. The potential region between -1.08 and $-0.470 \mathrm{~V}$ (nhe), pre-peak zone, is mainly characterized by a reversible process, at $-0.870 \mathrm{~V}$, overlapping and catalysing the her, ascribed to the formation of an $\mathrm{Ag}-\left(\mathrm{SH}^{-}\right)_{\text {ds }}$ bond. Phase $\mathrm{Ag}_{2} \mathrm{~S}$ deposits were found to fit a linear relationship between reflectance, in the blue region, and charge. The oxidation of surface $\mathrm{Ag}_{2} \mathrm{~S}$ (postwave) is readily observed by differential reflectance at potentials positive of the onset of bulk $\mathrm{Ag}_{2} \mathrm{~S}$ formation. The rough silver surface brought about by the reduction of $\mathrm{Ag}_{2} \mathrm{~S}$ allows a strong coupling between photons and surface plasmons.
\end{abstract}

Key words: thin films, sulphide electrodeposition, silver tarnisining, plasmons, electroreflectance.

\section{INTRODUCTION}

Silver sulphide films have been a subject of increasing interest in the last few years because of their electrical and optical properties[1-9]. The electroformation of silver sulphide films on silver metal is of interest for its application to ion selective electrodes [10] and, from the viewpoint of corrosion studies, in relation to the tarnishing phenomena of silver. The electrochemical recovery of silver from silver sulphide materials is also of industrial relevance. In addition, silver sulphide systems have been investigated with a view to developing superionic conductors[11] and photosensitive materials for recording media[12].

In this communication we present data, obtained through in situ optical (uv-visible) and electrochemical techniques, for relatively thin films of silver sulphide, not thicker than ca. $100 \AA$, grown on polycrystalline silver electrodes with the aim of providing complementary information on the properties of the film species.

\section{EXPERIMENTAL}

Optical measurements were obtained with $p$ polarized light at an incident angle of $59^{\circ}$ using either a reflectance spectrometer specially designed for electrochemical systems with the aid of potential modulation [13], or a computerized optical multichannel analyser (OMA) fitted with a cooled $\mathrm{Si}$ diode array. This rapid-scan spectrometer was employed to obtain two types of integral spectra, namely during slow linear potential sweeps, $c a$. $2 \mathrm{mVs}^{-1}$, every spectrum resulting from the coaddition in computer memory of 100 exposures, each averaged $0.03 \mathrm{~s}$ on the diode array chip and after a

- Author to whom correspondence should be addressed. potential step, monitoring the ensuing transient by means of successive spectra comprising of 50 exposures of $0.03 \mathrm{~s}$ each. Diffraction orders higher than one were sorted out by appropriate filters. Electrochemical experiments were carried out in a conventional way.

The working electrode was a Kel-F-encased silver disc (Specpure, Johnson Matthey), polished to a mirror finish with alumina and cleaned in an ultrasonic bath before being introduced into the deoxygenated supporting electrolyte. Solutions were prepared with redistilled Millipore water and pa. grade $\mathrm{Na}_{2} \mathrm{~S} .9 \mathrm{H}_{2} \mathrm{O}$ (Merck). The base electrolyte was a borate buffer (Merck pa.) of pH 9.2. Potentials were measured against a mercurous oxide reference electrode and are quoted on the normal hydrogen electrode scale.

\section{RESULTS AND DISCUSSION}

\subsection{Electrochemical characterization}

Conventional voltammetric runs were carried out to define the potential zones where the different processes develop and to determine the relationships that characterize their behaviour. The observed current response (Fig. 1) coincides on the whole with that reported by Hepel et al.[7] with the expected changes arising from the difference between the solution $\mathrm{pH}$ of the two studies. However, in addition to the optical information, further details are presented in the electrochemical data of this work, ie a postwave in the bulk deposition of silver sulphide, and the presence of peak $A_{1}$, emphasized as the most relevant in the pre-peak zone (vide infra). The voltammogram in Fig. 1 shows two main potential regions, the pre-peak region between -1.08 and $c a$. $-0.470 \mathrm{~V}$, partially overlapping the hydrogen evolution reaction (her), and the zone where the electrodeposition and reduction of phase silver sulphide take place. The pre-peak region is dominated by the 
process that takes place at $-0.870 \mathrm{~V}$ (described as $A_{1}$ below*) that overlaps and catalyses at her, leading to the presence of a dip, $C^{*}$, on the positivegoing scan, followed by a small and sharp peak $A_{2}$ at $-0.570 \mathrm{~V}$. The negative wave $C^{*}$, is also observed, with the same sign, on the back scan. This peculiar behaviour of $C^{*}$ could be explained by assuming the negative current (wave) arises from the her catalysed by the deposition of sulphide species $A_{1}$ at intermediate coverage[3]. The accumulated charge, up to $-0.470 \mathrm{~V}$, corresponding to both peaks $A_{1}$ and $A_{2}$, was estimated for $2 \times 10^{-4} \mathrm{M}$ sulphide, in steadystate conditions, by means of chronocoulometry fol-

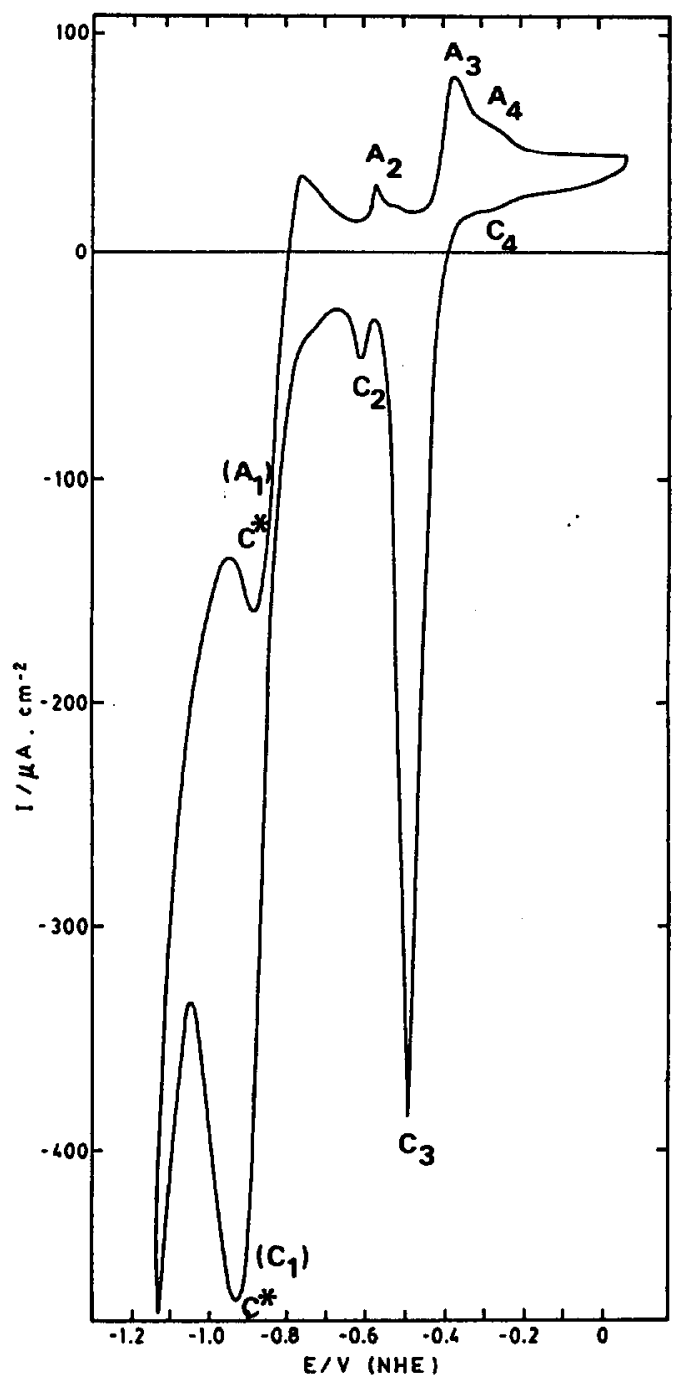

Fig. 1. Voltammogram run at $50 \mathrm{mVs}^{-1}$ (single scan), covering the pre-peak and bulk silver sulphide potential regions; $2.5 \times 10^{-4} \mathrm{M}$ sulphide in sodium tetraborate solution, pH 9.2.

* Process no. 1 is indicated as $C^{*}$ because it shows on the $c v$ as a negative current irrespective of the sweep slope, it arises however from the underlying surface reactions $A_{1} / A_{2}$. lowing the procedure detailed in [14] and found to be ca. $200 \pm 20 \mu \mathrm{C} \mathrm{cm}^{-2}$.

At potentials positive of $c a$. $-0.470 \mathrm{~V}$, the electroformation of the phase silver sulphide can be observed, $A_{3}$, under diffusion control, followed by a hump, $A_{4}$, superimposed on the falling current profile of peak $A_{3}$. The equilibrium potential of the sulphide/silver sulphide system was calculated by using the relationship reported in [4] and found to be $-0.436 \mathrm{~V}$ for the concentration employed in this work. An effective diffusion coefficient for $\mathrm{SH}^{-}$was calculated from a plot of charge vs. square root of time, the data being obtained from a potential step from -0.8 to $-0.1 \mathrm{~V}$. The value was found to be $1.19 \times 10^{-5} \mathrm{~cm}^{2} \mathrm{~s}^{-1}$. Current peak $A_{4}$ was found to prevail upon $A_{3}$ at high sweep rates since the former grows as $v$ instead of $v^{1 / 2}$. The wave $A_{4}$ is assigned to the oxidation of surface silver sulphide, its potential being more positive than $A_{3}$ on account of the higher stability of the surface species. On reversing the potential scan the postwave pair, $C_{4}$, can be obtained, followed by the electroreduction of silver sulphide, peak $C_{3}$, the profile being typical of a phase formation; that is, recrystallization of silver ions to give silver metal with the attending dissolution of sulphide ions. Under special experimental conditions, namely low sweep rates, $20 \mathrm{mV} \mathrm{s}^{-1}$, peak $C_{3}$ was found to split into two components. The complementary peaks $C_{2}$ and $C_{1}$ are more pronounced than on the forward scan, particularly $C_{1}$, which is also wider, probably due to the further reduction of species formed during the precedent scan. $C_{2}$ appears somewhat better resolved than $A_{2}$ because of the increase in surface roughness brought about by the process of formation/reduction of phase silver sulphide.

\subsection{Optical measurements}

3.2.1. Integral measurements. The system was studied by measuring the optical response to rather large potential perturbations, typically a few hundred millivolts.

Integral measurements, as a function of the wavelength, in the pre-peak region (Fig. 2a), exhibit a somewhat similar shape to that of phase silver sulphide films (Fig. 3). The absorption maximum corresponding to surface silver sulphide was found to be at $333 \mathrm{~nm}$ for thin films. However, $380 \mathrm{~nm}$ was selected for measurements in the blue region since it gave a more favourable signal/noise ratio due to the higher power of the quartz-halogen source at lower frequencies. Potentiostatic pulses were applied between -0.8 and $-0.1 \mathrm{~V}$, the optical response, $(R$ $\left.-\boldsymbol{R}_{0}\right) / \boldsymbol{R}_{0}$, as taken at $380 \mathrm{~nm}$ (Fig. 4), exhibits a linear relationship when plotted against $t^{1 / 2}$ indicating the change of reflectivity of silver, at the chosen wavelength, is proportional to the amount of silver sulphide laid down.

Figure 3 shows spectra taken at 3.0 -s intervals after a potential step between -0.65 and $-0.15 \mathrm{~V}$ has been applied. In addition to the $\mathrm{AgS}_{2}$ band at $339 \mathrm{~nm}$, an intense plasmon contribution is observed at ca. $420 \mathrm{~nm}$ that tails slowly into the red region. However, silver sulphide itself is known to absorb radiation throughout the visible region as its band gap is reported to be $0.9 \mathrm{eV}$ [15]. Nevertheless, higher 


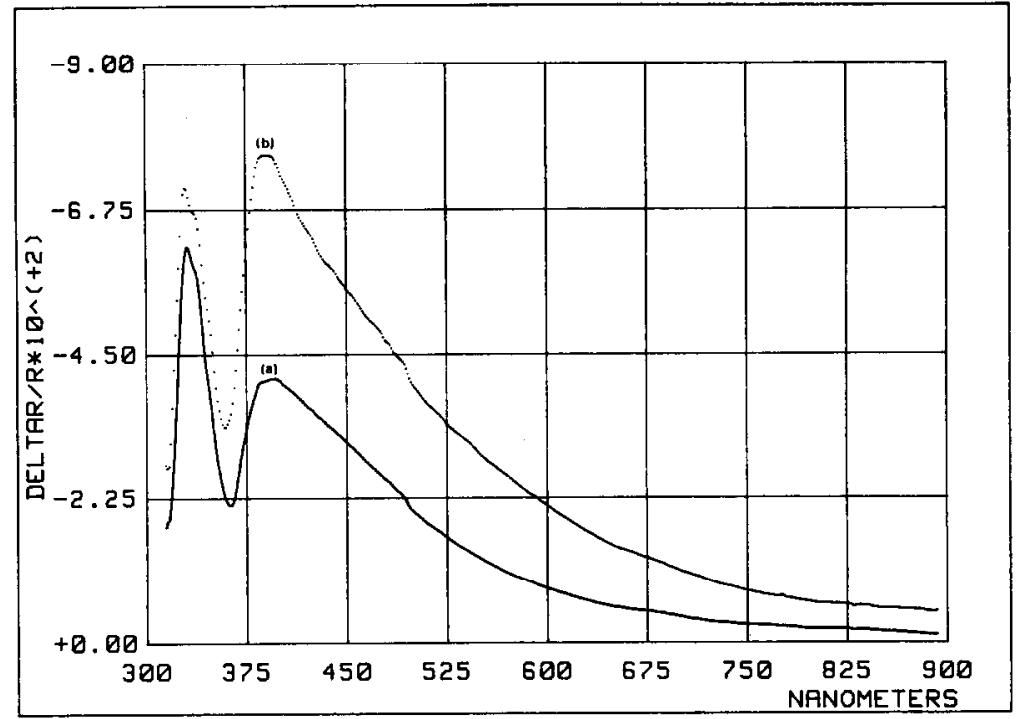

Fig. 2. Normalized integral reflectance measurements, $\Delta R / R$, as obtained by the difference between spectra at two given potentials. Spectra taken during scanning cyclically at $2 \mathrm{mV} \mathrm{s}^{-1}$ from -1.15 to $-0.15 \mathrm{~V}$. The individual reflectance spectra ( $R$ curves), at each potential, were 100 times averaged in memory, exposure time: $0.03 \mathrm{~s}$, that is, each spectrum took $3 \mathrm{~s}$, potentials quoted are starting values: p-polarized light, $\phi=59^{\circ} ; 2.5 \times 10^{-4} \mathrm{M}$ sulphide; pre-peak region; spectra smoothed out by the Savitzky-Golay algorithm: (a) $\Delta R / R=\left(R_{-0.73 \mathrm{v}}-R_{-1.10 \mathrm{v}}\right) / R_{-1.10 \mathrm{v}}$, forward scan; and (b) $\Delta R / R=$ $\left(R_{-0.77 \mathrm{v}}-R_{-1.10 \mathrm{v}}\right) / R_{-1.10 \mathrm{v}}$, back scan (the small break at $493 \mathrm{~nm}$ is an artifact).

energies for the band gap have been described for thin films[16].

On the other hand, the spectrum in the pre-peak region when observed on the back scan, after the reduction of phase silver sulphide, and compared with the spectrum taken on the forward scan for the same potential region, illustrate the relative enhancement of the surface plasmon excitation (Fig. 2b). This effect should likely arise from the increase in surface roughness. The behaviour is, on the whole, similar to the process of silver roughening in the presence of chloride ions by repetitive formation and reduction of $\mathrm{AgCl}$ which leads to the well-known effect of surface enhanced Raman[17].

In order to gain a finer insight into the reaction processes, not easily drawn from the broad features

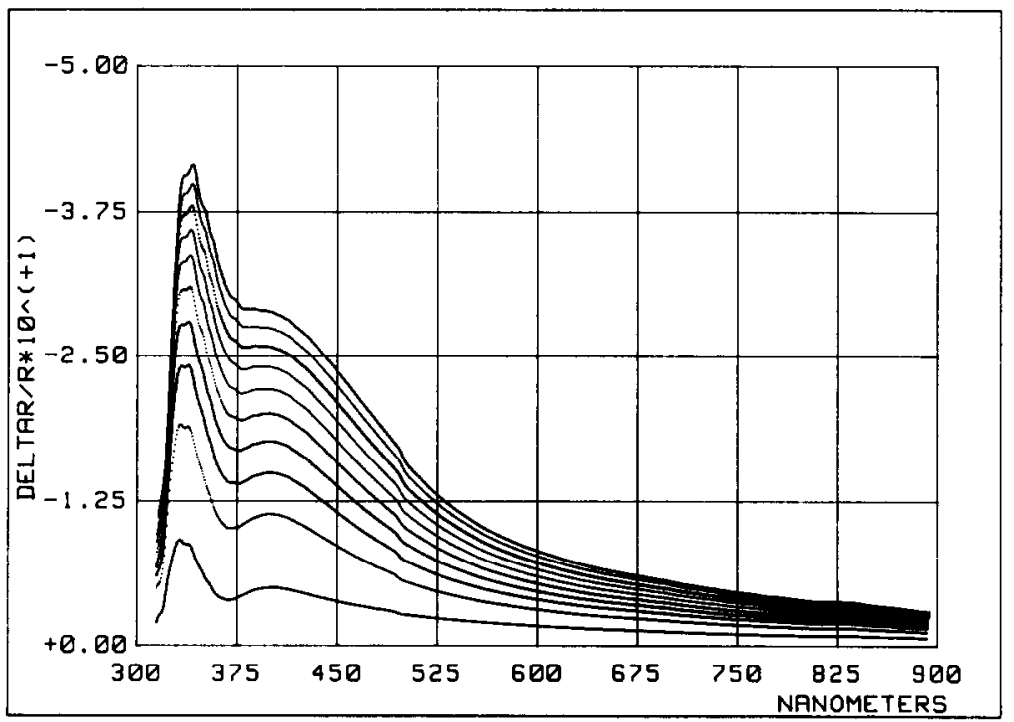

Fig. 3. Time dependence of the integral change of reflectance vs. wavelength, $\Delta R / R=\left(R_{-0.15 v}\right.$ $\left.-R_{-0.65 \mathrm{v}}\right) / \boldsymbol{R}_{-0.65 \mathrm{v}}$, resulting from the formation of bulk silver sulphide: $p$-polarized light, angle of incidence $\phi=59^{\circ}$; potential stepped from -0.65 to $-0.15 \mathrm{~V}$. Each spectrum, measured during the ensuing transient, was averaged 50 times in memory, exposure time: $0.03 \mathrm{~s}$, that is, each curve took $1.5 \mathrm{~s}$. In this figure, however, they are spaced out $3 \mathrm{~s}$ as only half of the total number of spectra (20) are shown. The final charge after $30 \mathrm{~s}$ was determined to be $c a .1500 \mu \mathrm{Cm}^{-2}$. 


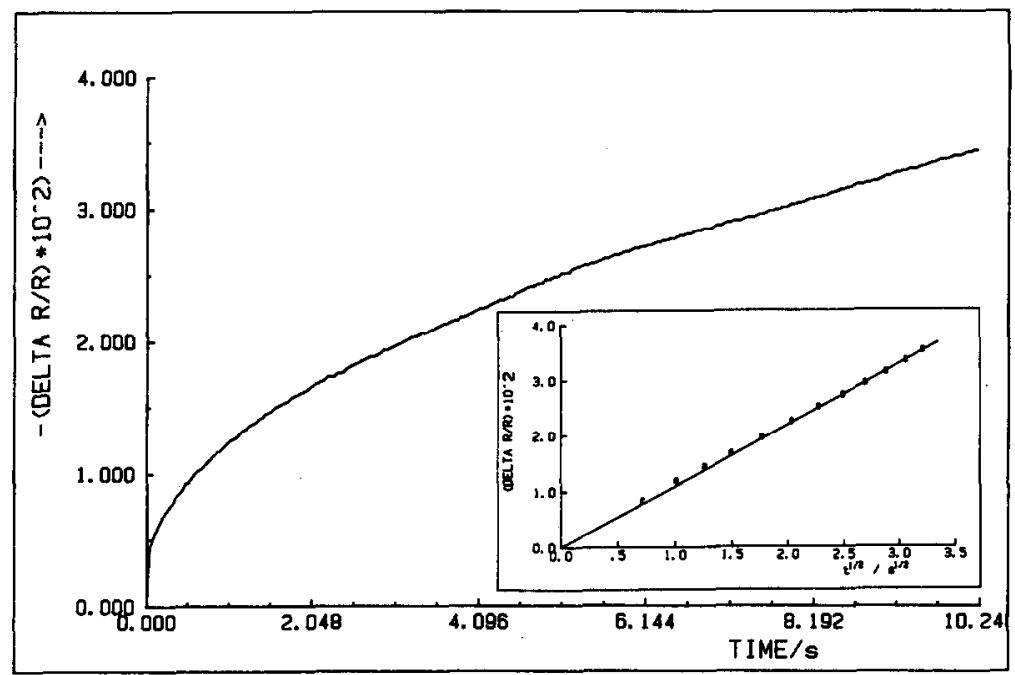

Fig. 4. Time dependence of the integral change of reflectance, $\Delta R / R$, attending the formation of bulk silver sulphide: potential stepped from -0.8 to $-0.1 \mathrm{~V}$; p-polarized light, angle of incidence $\phi=59^{\circ}$; $380 \mathrm{~nm}$. Inset: $\Delta R / R$ vs. $t^{1 / 2}$ relationship.

of the integral spectrum, differential measurements as a function of potential were carried out at set wavelengths.

3.2.2. Differential measurements. Reflectance determinations were carried out at fixed wavelengths, namely 380 and $680 \mathrm{~nm}$, by sweeping the potential linearly between -1.08 and $+0.02 \mathrm{~V}$ at $3.6 \mathrm{mVs}^{-1}$ with a superimposed $50 \mathrm{mV}$ amplitude modulation at $11 \mathrm{~Hz}$. The optical signal was demodulated by a lock-in amplifier and the rectified in-phase component plotted vs. potential as in Fig. 5. The curve taken at $380 \mathrm{~nm}$ shows (Fig. $5 \mathrm{a}$ ) on the forward scan, a large and broad maximum at $c a .-0.900 \mathrm{~V}$ in the region corresponding to the $C^{*}$ dip of the voltammogram attending the electroadsorption of sulphide species, in a relatively fast process when compared to the modulation frequency. The species giving rise to this peak was found to catalyse the hydrogen evolution reaction as has been reported by Parsons et al.[3]. Its presence has also been suggested by Horányi and Vertes [6]. The reaction involved in this process can be written, as has been proposed for gold electrodes $[14,18]$,

$$
\mathrm{Ag}+\mathrm{SH}^{-} \leftrightarrow \mathbf{A g}-\left(\mathbf{S H}^{-}\right)_{\text {ads }},
$$

leading to interacting surface species with a significant ionic character and enough kinetic reversibility so as to follow the potential modulation. Process $A_{2}$ is detected as a small, sharp peak on the falling optical response of reaction $A_{1}$. Under the pertur-

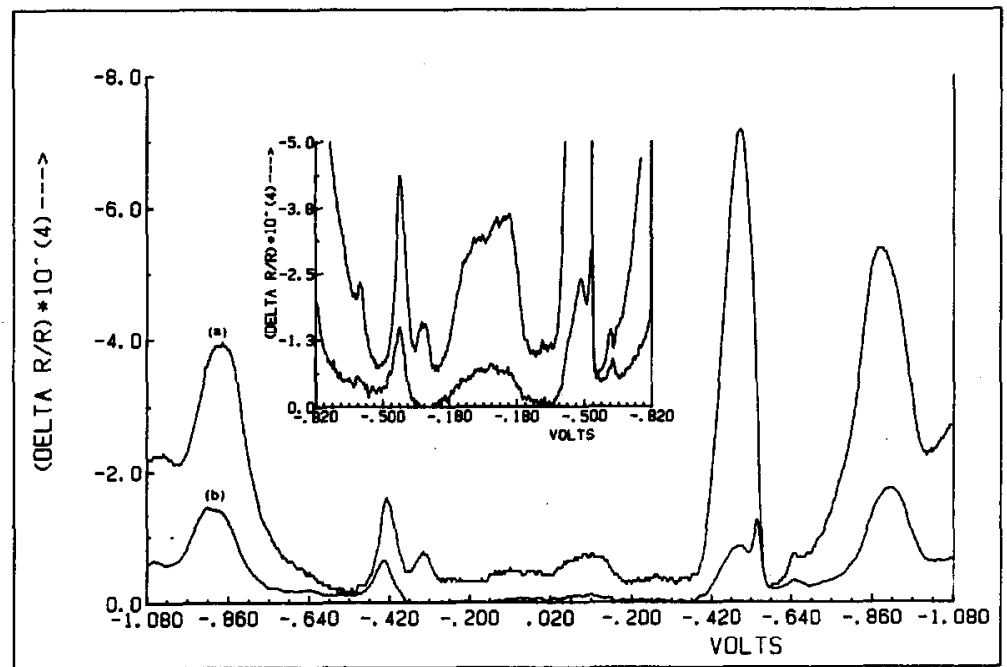

Fig. 5. Difierential reflectance, $\delta R / R$, vs. electrode potential curves: modulation amplitude $50 \mathrm{mV}$ $f=11 \mathrm{~Hz}$; potential was scanned cyclically from -1.080 to $+0.02 \mathrm{~V}$ at $3.6 \mathrm{mV} \mathrm{s}^{-1}$; p-polarized light, angle of incidence, $\phi=59^{\circ}$ : (a) $380 \mathrm{~nm}$; and (b) $680 \mathrm{~nm}$. Inset: $\delta R / R$ vs. electrode potential plot showing the region between -0.82 and $-0.20 \mathrm{~V}$, forward and back scans, sweep rate $2.6 \mathrm{mV} \mathrm{s}^{-1}$. Other conditions as in main graph. 
bation applied the process $A_{2} / C_{2}$ appears as poorly reversible, that is, does not seem to follow the modulation. The transformation associated with these maxima gives rise to a strongly surface species, probably attended by deprotonation, with similar properties to a two-dimensional silver sulphide that can be optically detected at both 380 and $680 \mathrm{~nm}$.

As the potential is made more positive a maximum can be observed corresponding to the formation of phase silver sulphide under diffusion control, identified as $A_{3}$ on the $c v$, followed by a peak revealing the presence of postwave $A_{4}$, this process is detected at 380 , but not at $680 \mathrm{~nm}$; this fact points to the different optical properties of the underlying thin layer in comparison with that of the bulk silver sulphide deposit. The subsequent slanting profile, even after the potential reversal, arising from the growth of phase silver sulphide, its reduction being noticed at $-0.500 \mathrm{~V}$ on the back scan. Peak $C_{4}$ does not show up in the optical profile. The tiny signal at $-0.635 \mathrm{~V}$ points to the modulation of process $C_{2}$, better resolved on the reverse sweep than on the forward scan because of an increase in surface roughness. A similar enhancement in magnitude is observed for the peak at $-0.915 \mathrm{~V}$, on the negative-going scan, arising from the change of electrode area. On the other hand, when similar measurements are carried out at $680 \mathrm{~nm}$ (Fig. 5b) in addition to having a much weaker signal, there are some differences to be noted, namely, the maximum corresponding to reaction $A_{4}$ is absent, as described earlier. On the negative-going scan, the most interesting feature is observed in the reduction region of silver sulphide, the trailing edge of the maximum is characterized by the presence of a sharp peak. This behaviour was found to be reproducible and clearly detected. The spectrum of phase silver sulphide, as grown under our experimental conditions, reveals a significant contribution from the excitation of surface plasmons (vide supra). In the potential region where this peak shows up, the silver surface should be partially covered by silver sulphide patches not yet reduced. Besides, the freshly reformed silver layer has got a rough and porous structure $[4,5]$. Thus, the spike observed may come from the excitation of surface plasmons as a consequence of the experimental conditions developed in the process of silver sulphide reduction. That is, the coupling between light and metal may take place through the rough silver surface reformed during silver sulphide dissolution[19]. Another possibility is that the silver sulphide irregular layer could act as a coupler for the light in a way similar to that described in [20] for pyridine films.

Acknowledgements-This work was supported by grants from the Consejo Nacional de Investigaciones Cientificas y Técnicas and the Comisión de Investigaciones Cientificas de la Provincia de Buenos Aires.

\section{REFERENCES}

1. J. L. Stanford, J. Opt. Soc. Am. 60, 49 (1970).

2. J. M. Bennett, J. L. Stanford and E. J. Ashley, J. Opt. Soc. Am. 60, 224 (1970).

3. G. Nguyen Van Huong, R. Parsons, P. Marcus, S. Montes and J. Oudar, J. electroanal. Chem. 119, 137 (1981).

4. V. I. Birss and G. A. Wright, Electrochim. Acta 26, 1809 (1981).

5. V. I. Birss and G. A. Wright, Electrochim. Acta 27, 1 (1982).

6. G. Horányi and G. Vértes, Electrochim. Acta 31, 1663 (1986).

7. M. Hepel, S. Bruckenstein and G. C. Tang, J. electroanal. Chem. 261, 389 (1989).

8. H. Haefke, A. Panov and V. Dimov, Thin Solid Films 188,133 (1990).

9. K. Bozhilov, V. Dimov, A. Panov and H. Haefke, Thin Solid Films 190, 129 (1990).

10. J. B. Jensen, Anal. Chim. Acta 76, 279 (1975).

11. T. Minami, J. Non-Cryst. Solids $95-96,107$ (1987).

12. A. Kinoshita, Jpn. J. appl. Phys. 13, 1027 (1974).

13. R. O. Lezna, N. R. de Tacconi, J. A. Rapallini and A. J. Arvia, An. Asoc. Quim. Argent. 76, 25 (1988).

14. R. O. Lezna, N. R. de Tacconi and A. J. Arvia, J. electroanal. Chem. 283, 319 (1990).

15. C. Rao and K. Pisharody, Prog. Sol. State Chem. 10, 207 (1976).

16. A. J. Varkey, Int. J. mat. prod. Tech. S, 264 (1990).

17. M. Fleischmann, P. J. Hendra, A. J. McQuillan, R. L. Paul and E. S. Reid, J. Raman Spect. 4, 269 (1976).

18. X. Gao, Y. Zhang and M. J. Weaver, Langmuir 8, 668 (1992).

19. E. A. Stern, in Optical Properties and Electronic Structures of Metals and Alloys, (Edited by F. Abelès), p. 396. North-Holland, Amsterdam (1966).

20. T. López-Ríos and Y. Borensztein, Surf. Sci. 226, 131 (1990). 